\title{
Analysis of the Electro-hydraulic Steering System of the Wheel Construction Machinery
}

\author{
Dong Li \\ Xi'an Aeronautical Polytechnic Institute, Shaanxi 710089, China \\ lidongxhy@qq.com
}

Keywords: List the keywords covered in your paper. These keywords will also be used by the publisher to produce a keyword index.

\begin{abstract}
This template explains and demonstrates how to prepare your camera-ready paper for Trans Tech Publications. The best is to read these instructions and follow the outline of this text. Please make the page settings of your word processor to A4 format ( $21 \times 29,7 \mathrm{~cm}$ or $8 \times 11$ inches); with the margins: bottom $1.5 \mathrm{~cm}$ (0.59 in) and top $2.5 \mathrm{~cm}(0.98 \mathrm{in})$, right/left margins must be $2 \mathrm{~cm}$ ( 0.78 in). Your manuscript will be reduced by approximately $20 \%$ by the publisher. Please keep this in mind when designing your figures and tables etc.
\end{abstract}

\section{Introduction}

The wheel construction machinery shows strong environmental adaptability and favorable maneuvering performance, when being applied to the practical application process. Along with development of society, there has been a higher requirement of the operational efficiency, which has in turn promoted automation of the construction machinery. Meanwhile, there is a higher expectation of the system's control performance. This paper mainly analyzes and studies the electro-hydraulic steering system of the wheel construction machinery.

Please keep a second copy of your manuscript in your office. When receiving the paper, we assume that the corresponding authors grant us the copyright to use the paper for the book or journal in question. Should authors use tables or figures from other Publications, they must ask the corresponding publishers to grant them the right to publish this material in their paper.

Use italic for emphasizing a word or phrase. Do not use boldface typing or capital letters except for section headings (cf. remarks on section headings, below).

\section{The system}

The steering system plays an important role in a machine. Researchers have widely investigated into how to overcome defects of the transmission system during the application process. The fruitful research findings have led to emergence of the electric control power-assisted steering, electric power steering and hydraulic power-assisted steering. Among them, the application advantages of the electro-hydraulic steering are particularly outstanding. To deepen research into the research holds vital significance to its further applications.

\subsection{Overview of the wheel construction machinery electro-hydraulic steering system}

The wheel construction machinery electro-hydraulic steering system is a control system jointly made up of the energy exchange elements and the hydraulic control elements. Here, the energy exchange elements and the hydraulic control elements include the hydraulic pump for manufacturing, hydraulic control valve, etc. The hydraulic control technology is critical to the automatic control technology. During the application process, the hydraulic control system has exhibited outstanding advantages and striking characteristics. For example, it allows a large area of machine-free speed adjustment during the application process; its movement during the gapless transmission process is 
quite steady; under the status of equivalent power transmission, the hydraulic transmission device is highly dynamic, weight, and long in service life; the hydraulic elements are capable of self-adjustment, etc. However, the hydraulic control technology falls under the scope of the typical electromechanical hydraulic integrated technology, which involves a large range of fields [1].

\subsection{Research into the wheel construction machinery electro-hydraulic steering system design}

\subsubsection{Design principle of the machinery electro-hydraulic steering system}

The system features the horizontal dual-pull rod hydraulic cylinder. Meanwhile, the handle block sliding structure is employed. The pressure oil is controlled by the proportional valve, which serves to activate the movement of the piston. Under the pushing effect of the connecting rod, the steering wheel realizes its deflection and finish the steering of the whole machine. The following schematic diagram shows the work principle of the electro-hydraulic steering valve:

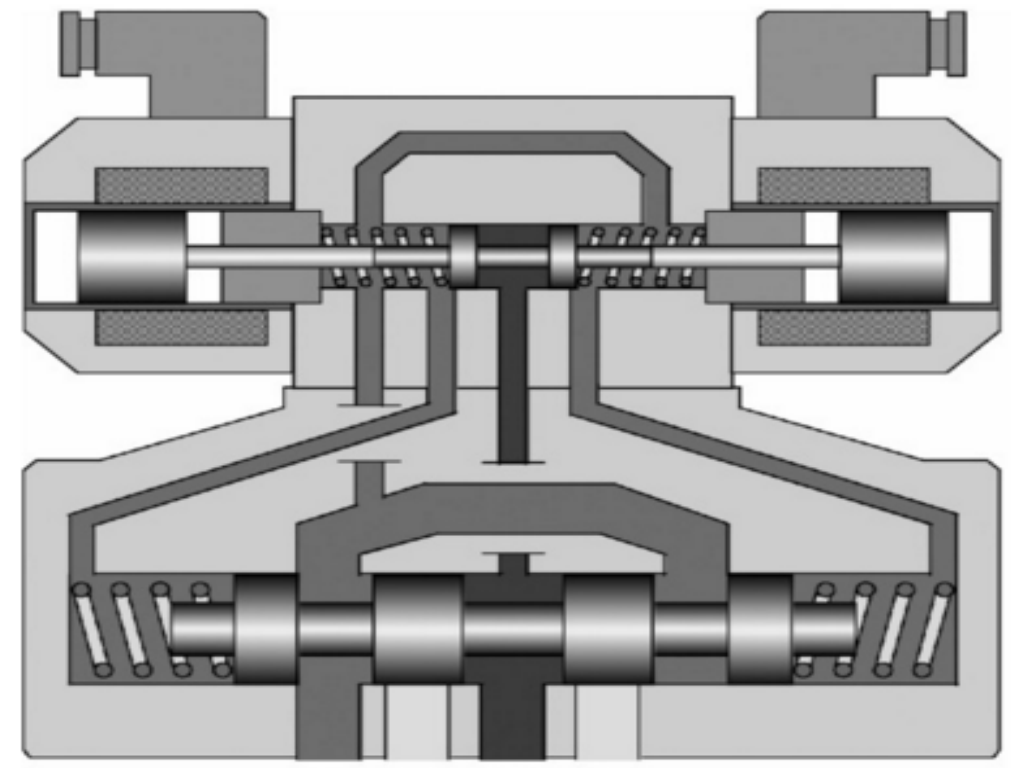

Fig. 1 Before steering of the hydro-electric steering valve

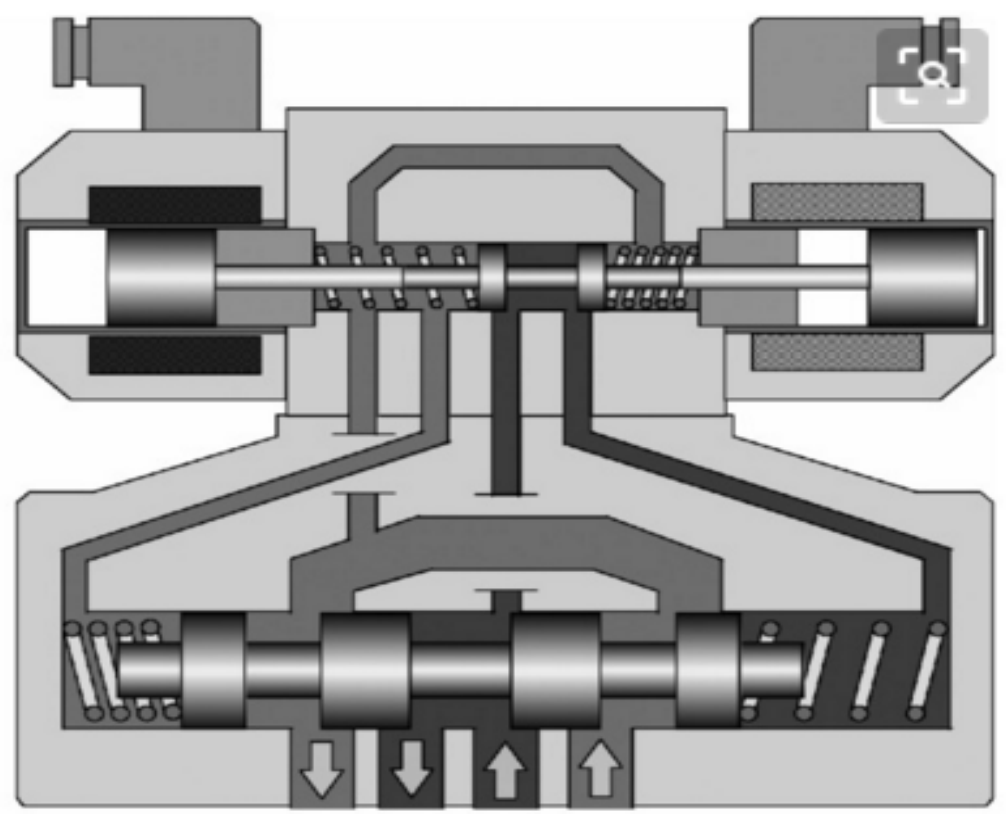

Fig. 2 After steering of the hydro-electric steering valve 
During the specific application process, the structure mainly adopts the dual feeler level hydraulic cylinder as the steering performing structure. The piston rod serves as the horizontal pull rod. During the process, the steering style mechanism and the steering hydraulic cylinder are integrated into a whole to realize the purpose of simplifying the structure. Meanwhile, a series of advantages is thus obtained, including a convenient layout, a compact structure, the same sensitivity, and consistency between the left and right side. However, it also has a defect, that is, during the practical steering process, the hydraulic cylinder has to undertake the large radial force. In order to alleviate the problem, the trapezoid optimization design (See Fig. 3) is adopted for the process of parameter selection. In this way, reasonability and preciseness of various parameters can be ensured.

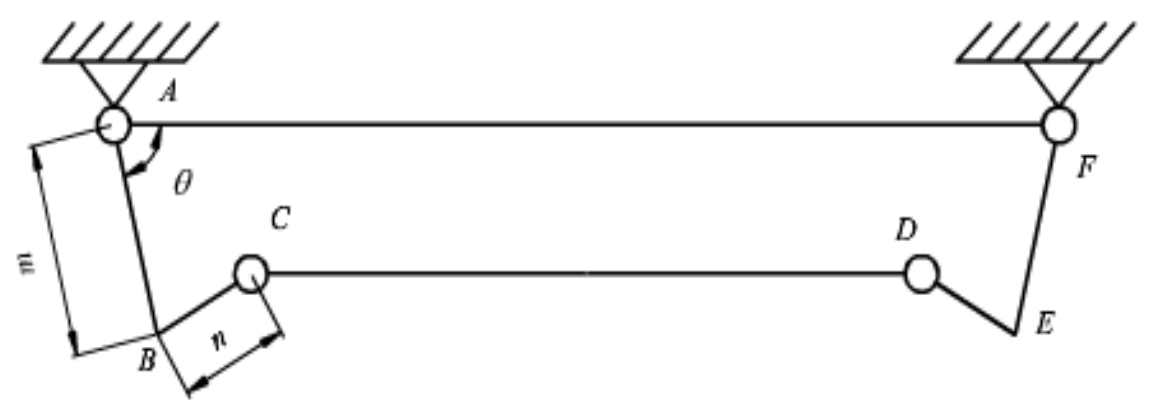

Fig. 3 Schematic diagram of the steering trapezoid structure

\subsubsection{Design requirements of the machinery electro-hydraulic steering system}

During the process of the steering system design, the maneuvering characteristics of the machine should be maintained to be favorable. Under the limited site conditions, the machine should be able to make small turns. Below are specific requirements.

First, restricted by the construction machinery conditions, the machine often has to drive on rugged roads. Hence, there is a higher requirement of the service life and strength of the steering structure and parts. Meanwhile, the reliability and safety of the structure should be guaranteed. Second, the machinery operation is usually frequent. Therefore, the machine should be made easier for drivers so as to improve working efficiency. Third, in order to alleviate the driving resistance, previous of the machinery movement traces and driving direction should be ensured. In addition, tire abrasion should be minimized. All wheels should revolve around the same clockwise rotation and steer to the center. Fourth, maintenance of the steering mechanism should be convenient. The structural simplification and adjustment are necessary.

\subsubsection{Optimized design of the steering trapezoid structure}

This is a critical link in the machinery steering mechanism. During the process, the binding parameters of the trapezoid structure should be confirmed so that the ideal steering angle of the inner and outer steering wheels can be guaranteed. The steering style arm length, trapezoid bottom angle and other factors of the trapezoid mechanism have a close bearing on the steering performance of the car. All these should arouse attention from designers [2].

In order to ensure smoothness of the car, reduce the steering resistance and avoid occurrence of the wheel abrasion, all wheels are required to have pure rolling, namely the "theoretical steering style". Under the condition, the relationship between the vehicle inner and outer steering angle can be expressed by the following formula:

$$
\operatorname{ctg} \alpha=\operatorname{ctg} \beta+\frac{K}{L}
$$


Where, $\alpha$ denotes the steering angle of the front outer wheel; $\beta$ denotes the steering angle of the front inner wheel; K denotes the front axle swizzle center distance; $\mathrm{L}$ denotes the wheel base between the front axle and the back axle. According to the above situations, the idealized $\alpha$ can be written as below:

$$
\alpha=\operatorname{arcctg}\left(\operatorname{ctg} \beta+\frac{K}{L}\right)
$$

During the calculation process, the linear drawing instructions provided by MATLAB and the relevant function resources can be adopted for solution. The function relationship that the outer steering wheel rotates along with the inner steering wheel under the ideal status is shown in Table 1.

Table 1. Theoretical angle of deflection

\begin{tabular}{|c|c|c|c|c|c|c|}
\hline $\begin{array}{l}\text { Theoretical } \\
\text { inner steering angle } \\
\qquad(\beta)\end{array}$ & 0 & 5 & 10 & 15 & 20 & 25 \\
\hline $\begin{array}{c}\text { Theoretical } \\
\text { outer steering angle } \\
(\alpha)\end{array}$ & 0 & 4.78 & 9.22 & 13.33 & 17.15 & 27.45 \\
\hline
\end{tabular}

The combination method is utilized for deviation of relevant formulas. Meanwhile, the design parameters are introduced. Finally, the steering angle of the steering mechanism designed for the electro-hydraulic steering system can be obtained. Besides, since the left and right steering structures are fully symmetric, only the right steering conditions should be analyzed.

During the design process, the outer steering angle corresponding to every inner steering angle is tabulated, such as Table 2.

Table 2. Outer steering angle of the inner steering angle

\begin{tabular}{ccccccc}
\hline $\begin{array}{c}\text { Theoretica } \\
\text { l inner } \\
\text { steering angle }\end{array}$ & 0 & 2 & 4 & 6 & 8 & 10 \\
\hline $\begin{array}{c}\text { Theoretica } \\
\text { 1 steering } \\
\text { angle }\end{array}$ & 0 & 1.98 & 3.68 & 5.8 & 7.45 & 9.22 \\
$\begin{array}{c}\text { Designed } \\
\text { inner steering } \\
\text { angle }\end{array}$ & 0 & 2 & 4 & 6 & 8 & 10 \\
$\begin{array}{c}\text { Designed } \\
\text { outer steering } \\
\text { angle } \\
\text { Absolute } \\
\text { deviation }\end{array}$ & 0 & 2.00 & 4.05 & 6.08 & 8.15 & 10.25 \\
\hline
\end{tabular}

In fact, during the specific design process, the steering mechanism can hardly satiate the relational expression between the inner steering angle and the outer steering angle, and the consistency between the two cannot be fully precise but just close to preciseness. As along as the consistency is within a reasonable scope, the basic driving requirement can be satisfied. In this design, the comparison between the newly-designed steering angle and the theoretical inner and outer steering angle can be tabulated as below: 
Table 3. Comparison between the theoretical inner and outer steering angle and the newly-designed inner and outer steering angle

\begin{tabular}{ccccccc}
\hline $\begin{array}{c}\text { Theoretical } \\
\text { inner steering } \\
\text { angle }\end{array}$ & 0 & 5 & 10 & 15 & 20 & 25 \\
\hline $\begin{array}{c}\text { Theoretical } \\
\text { inner }\end{array}$ & 0 & 4.98 & 9.17 & 13.20 & 16.99 & 20.50 \\
$\begin{array}{c}\text { Newly-design } \\
\text { ed inner steering } \\
\text { angle }\end{array}$ & 0 & 5 & 10 & 15 & 20 & 25 \\
$\begin{array}{c}\text { Newly-design } \\
\text { ed outer steering } \\
\text { angle } \\
\begin{array}{c}\text { Absolute } \\
\text { deviation }\end{array}\end{array}$ & 0 & 4.78 & 9.18 & 13.13 & 16.58 & 19.40 \\
\hline
\end{tabular}

All in all, during the design process, SolidWorks can be used for improvement and optimization of the steering axle so as to reduce the stress of the trapezoid pull rod as much as possible, and increase the application intensity of the trapezoid arms as well. Meanwhile, the overall structure of the trapezoid arms can be further fastened, and their flexibility and reliability can be improved to avoid occurrence of vehicle abrasion and effectively extend the service life of the machine [3]. Additionally, to upgrade the practical performance of the control system, technical personnel should first clarify the basic performance indexes shown as below:

Table 4. Performance indexes of the control system

\begin{tabular}{ccccc}
\hline & Delay time & Rise time & Peak time & $\begin{array}{c}\text { Adjustment } \\
\text { time }\end{array}$ \\
\hline 10Mpa & 0.15 & 0.25 & 0.45 & 1.45 \\
$12 \mathrm{Mpa}$ & 0.155 & 0.26 & 0.410 & 1.45 \\
$14 \mathrm{Mpa}$ & 0.155 & 0.250 & 0.40 & 0.88 \\
\hline
\end{tabular}

As one observes in Table 4, as the system pressure keeps on increasing, the system response also accelerates, meaning that the response performance is improving. In this design, during the measurement process of the system steering performance, the relational expression between the steering angle and the displacement is often involved so as to maintain consistency of the mechanism parameters. Based on the relational expression, the corresponding steering angle of the hydraulic cylinder displacement can be obtained. Following that, the specific steering characteristic curve can be drawn.

\section{Conclusions}

To sum up, this paper briefly summarizes the wheel construction machinery electro-hydraulic steering system, and focuses on studying the system design. It is found out that the wheel construction machinery has the advantages of strong adaptability and favorable maneuverability. These are major causes of its wide applications in recent years. Under the condition, a higher requirement is raised of the machinery electro-hydraulic steering system, which requires more in-depth research by the future technical personnel.

\section{References}

[1] Ma L Y, Liang N X, Cao Y W, et al. Research on Test and Simulation of New Type Steering System for Construction Machinery[J]. Applied Mechanics \& Materials, 2013, 241-244(3):1974-1977. 
[2] Chen Y, Chang L, Xia J L. Energy consumption analysis of electro-hydraulic power steering system based on hardware-in-loop simulation test.[J]. Transactions of the Chinese Society of Agricultural Engineering, 2010, volume 26(8):117-122(6).

[3] Min Ye, Quan Wang, Shengjie Jiao. Robust, Control for the Electrohydraulic Steering System of a Four-Wheel Vehicle[J]. 2014, 2014(4):1-12.

[4] Ye M, Wang Q, Jiao S. Robust H 2 / H $\infty$ Control for the Electrohydraulic Steering System of a Four-Wheel Vehicle[J]. Mathematical Problems in Engineering, 2014, (2014-7-20), 2014, 2014(4):1-12. 